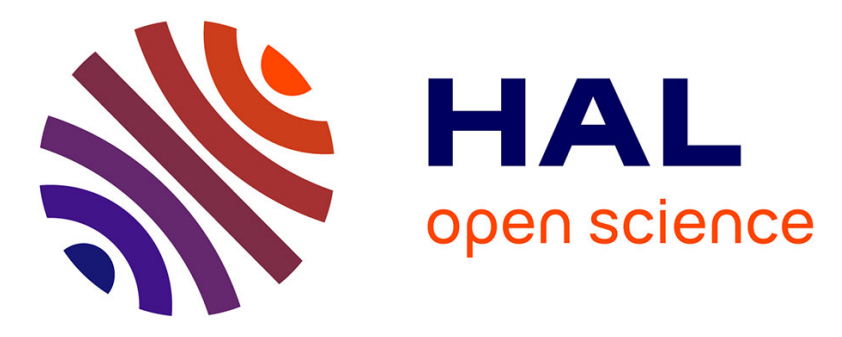

\title{
Pressure promoted low-temperature melting of metal-organic frameworks
}

Remo N Widmer, Giulio I Lampronti, Simone Anzellini, Romain Gaillac, Stefan Farsang, Chao Zhou, Ana Belenguer, Craig Wilson, Hannah Palmer, Annette Kleppe, et al.

\section{To cite this version:}

Remo N Widmer, Giulio I Lampronti, Simone Anzellini, Romain Gaillac, Stefan Farsang, et al.. Pressure promoted low-temperature melting of metal-organic frameworks. Nature Materials, 2019, 18 (4), pp.370-376. 10.1038/s41563-019-0317-4 . hal-02168638

\section{HAL Id: hal-02168638 https://hal.science/hal-02168638}

Submitted on 28 Jun 2019

HAL is a multi-disciplinary open access archive for the deposit and dissemination of scientific research documents, whether they are published or not. The documents may come from teaching and research institutions in France or abroad, or from public or private research centers.
L'archive ouverte pluridisciplinaire HAL, est destinée au dépôt et à la diffusion de documents scientifiques de niveau recherche, publiés ou non, émanant des établissements d'enseignement et de recherche français ou étrangers, des laboratoires publics ou privés. 


\section{Pressure Promoted Low-Temperature Melting of Metal-}

\section{Organic Frameworks}

Remo N. Widmer ${ }^{1}$, Giulio I. Lampronti ${ }^{1}$, Simone Anzellini ${ }^{2}$, Romain Gaillac ${ }^{3}$, Stefan Farsang ${ }^{1}$, Chao Zhou ${ }^{4}$, Ana M. Belenguer ${ }^{5}$, Craig Wilson ${ }^{6}$, Hannah Palmer ${ }^{7}$, Annette K. Kleppe ${ }^{2}$, Michael T. Wharmby ${ }^{2,8}$, Xiao Yu, ${ }^{9}$ Seth M. Cohen, ${ }^{9}$ Shane G. Telfer, $^{10}$ Simon A. T. Redfern ${ }^{1}$, François-Xavier Coudert ${ }^{3}$, Simon G. MacLeod ${ }^{6,11}$, and Thomas D. Bennett*7

${ }^{1}$ Department of Earth Sciences, University of Cambridge, Downing Street, Cambridge CB2 3EQ, UK.

${ }^{2}$ Diamond Light Source Ltd, Harwell Science and Innovation Campus, Didcot, OX11 ODE, UK.

${ }^{3}$ Chimie ParisTech, PSL University, CNRS, Institut de Recherche de Chimie Paris, 75005 Paris, France.

${ }^{4}$ Department of Chemistry and Bioscience, Aalborg University, DK-9220 Aalborg, Denmark.

${ }^{5}$ Department of Chemistry, University of Cambridge, Lensfield Road, Cambridge CB2 1EW, UK.

${ }^{6}$ Atomic Weapons Establishment, Aldermaston, Reading, RG7 4PR, UK.

${ }^{7}$ Department of Materials Sciences \& Metallurgy, University of Cambridge, 27 Charles Babbage Road, Cambridge CB3 OFS, UK

${ }^{8}$ Deutsches Elektronen-Synchrotron (DESY), Notkestrasse 85, 22607 Hamburg, Germany

${ }^{9}$ Department of Chemistry and Biochemistry, University of California, San Diego, La Jolla, California 92023-0358, United States

${ }^{10}$ MacDiarmid Institute for Advanced Materials and Nanotechnology, Institute of Fundamental Sciences, Massey University, Palmerston North 4442, New Zealand

${ }^{11}$ SUPA, School of Physics \& Astronomy, and Centre for Science at Extreme Conditions, The University of Edinburgh, Edinburgh, EH9 3FD, UK.

Email: tdb35@cam.ac.uk 


\section{Summary}

Metal-organic frameworks (MOFs) are microporous materials with huge potential for chemical processes, including retention or separation of guest molecules.

Structural collapse at high-pressure, and transitions to liquid states at high temperature, have recently been observed in this family. Here, we show that the effect of simultaneous high pressure and temperature application on ZIF-62 and ZIF-4 results in complex behaviour, with distinct high- and low-density amorphous phases occurring over different regions of the pressure-temperature phase diagram. In-situ powder X-ray diffraction, Raman spectroscopy and optical microscopy reveal that the stability of the liquid MOF-state expands significantly towards lower temperatures at intermediate, industrially achievable pressures. Furthermore, the MOF-glass formed by melt quenching the high temperature liquid is shown to demonstrate permanent, accessible porosity. Our results thus imply a novel route to the synthesis of functional MOF glasses at low temperatures, avoiding decomposition upon heating at ambient pressure. 


\section{Introduction}

The discovery of a growing family of over 60,000 metal-organic framework (MOF) and coordination polymer compounds ${ }^{1}$ demonstrates the chemical versatility of these functional microporous materials. Their high surface areas, generated by regular arrays of metal nodes connected by organic ligands, has led to proposed applications in water harvesting ${ }^{2}$, gas storage ${ }^{3,4}$, gas separation ${ }^{5}$ and catalysis ${ }^{6}$. Research in this field has focused predominantly on ordered, crystalline MOF materials. However, there is growing recognition of other unusual physical properties in this family of "soft crystals" flexibility ${ }^{9}$ and disorder ${ }^{10}$ extending our appreciation of the impact of MOF materials ${ }^{11,12}$. A rich set of structural responses to external forces has been uncovered ${ }^{13}$, including negative gas adsorption ${ }^{14}$, breathing ${ }^{15}$, and temperature $(T)^{-16}$ or pressure $(P)$-induced phase transitions ${ }^{17}$. However, much still remains to be discovered about MOFs, especially with respect to their dynamic behavior.

Like MOFs, silicates and other minerals also display a wide diversity of interesting physical behaviours, in particular at non-ambient conditions. For example, studies of stability boundaries and properties in $P$-T-space are well established ${ }^{18}$. Silica $\left[\mathrm{SiO}_{2}\right]$ itself is an archetypical material and shows seven crystalline polymorphs ${ }^{19,20}$, numerous metastable porous phases (all-silica zeolites), two polyamorphs ${ }^{21}$, and a complex melting curve at high- $P-T$ conditions. Surprisingly, despite their apparent chemical differences, many MOFs find analogues in the mineral world ${ }^{22}$. For example, certain metal-oxalate MOFs ${ }^{23,24}$ were recently shown to be synthetic analogues of two minerals ${ }^{25}$, stepanovite $\left[\mathrm{NaMgFe}\left(\mathrm{C}_{2} \mathrm{O}_{4}\right)_{3} \cdot 8-9 \mathrm{H}_{2} \mathrm{O}\right]$ and zhemchuzhnikovite $\left[\mathrm{NaMg}\left(\mathrm{Fe}_{0.4} \mathrm{Al}_{0.6}\right)\left(\mathrm{C}_{2} \mathrm{O}_{4}\right)_{3} \cdot 8-9 \mathrm{H}_{2} \mathrm{O}\right]$. 
More similarities between MOFs and minerals are apparent in terms of topological structure. Zeolitic imidazolate frameworks (ZIFs), an important subgroup of MOFs ${ }^{26}$, comprise tetrahedral $\mathrm{ZnN}_{4}$ units as their main building blocks. This structural motif, interlinked with imidazolate ( $\left.\mathrm{Im}, \mathrm{C}_{3} \mathrm{H}_{3} \mathrm{~N}_{2}^{-}\right)$based ligands, forms continuous lowdensity networks. ZIFs can be directly compared to natural zeolites, which form frameworks of interlinked tetrahedral $\mathrm{SiO}_{4}$ and $\mathrm{AIO}_{4}$ units. Both, $\mathrm{ZIFs}^{27,28}$ and zeolites $^{29,30}$ retain their microporosity even at relatively high $T$ and $P$. Even higher $T$ and $P$ ultimately induces phase transitions ${ }^{31-35}$ and melting ${ }^{36}$. The formation of glasses from such MOF-liquids ${ }^{37}$ is of special interest due to their nature as a new class of melt-quenched glass material formed of amorphous $\mathrm{SiO}_{2}$-like continuous random networks ${ }^{38}$. The formation of MOF glasses via solid state conversion or meltquenching is of great importance due to their inherent MOF-like chemical connectivity and composition. ${ }^{36,38}$ The glass formed from one structure, ZIF-62, has been demonstrated to exhibit significant resistance against crystallization ${ }^{39}$, whilst that formed from ZIF-76 has recently been demonstrated to show permanent, accessible porosity ${ }^{40}$. Alongside their unique internal structures, MOF glasses and liquids are of great interest since they provide a route to manufacturing complex, monolithic glassy MOF objects from MOF powders by melt-casting ${ }^{36}$ and hotpressing $^{41}$

The separate pressure and temperature dependent studies carried out on ZIFs previously, alongside the investigation of the behavior of the liquid MOF phase at higher pressures, motivated us to attempt to construct the first high P-T phase diagram of any metal-organic framework system. We selected ZIF-62 $\left[\mathrm{Zn}(\mathrm{Im})_{1.75}(\mathrm{blm})_{0.25}\right]$ (blm, benzimidazolate, $\left.\mathrm{C}_{7} \mathrm{H}_{5} \mathrm{~N}_{2}{ }^{-}\right)$due to its known stable liquid 
state, which occurs between the relatively low melting temperature of ca. $430^{\circ} \mathrm{C}$, and a decomposition temperature of ca. $500{ }^{\circ} \mathrm{C}^{36}$. The three-dimensional structure (Figure 1a and 1b) crystallizes under solvothermal conditions in the Pbca space group, and adopts a network topology identical to that of the phosphate minerals variscite ${ }^{42}$ and strengite ${ }^{43}$, and $\mathrm{CaGa}_{2} \mathrm{O}_{4}{ }^{44}$. Both the natural phosphates and the synthetic gallate display interesting pressure-induced polymorphism ${ }^{43,45}$. To map out the $P$-T phase diagram of ZIF-62, we combined in-situ powder X-ray diffraction (PXRD, Diamond Light Source, beamline I15) and optical microscopy at simultaneous high- $P$ and high- $T$ with ex-situ electron microscopy. We also performed first-principles constant-pressure molecular dynamics simulations in order to provide insight into the pressure dependence at the molecular level. 

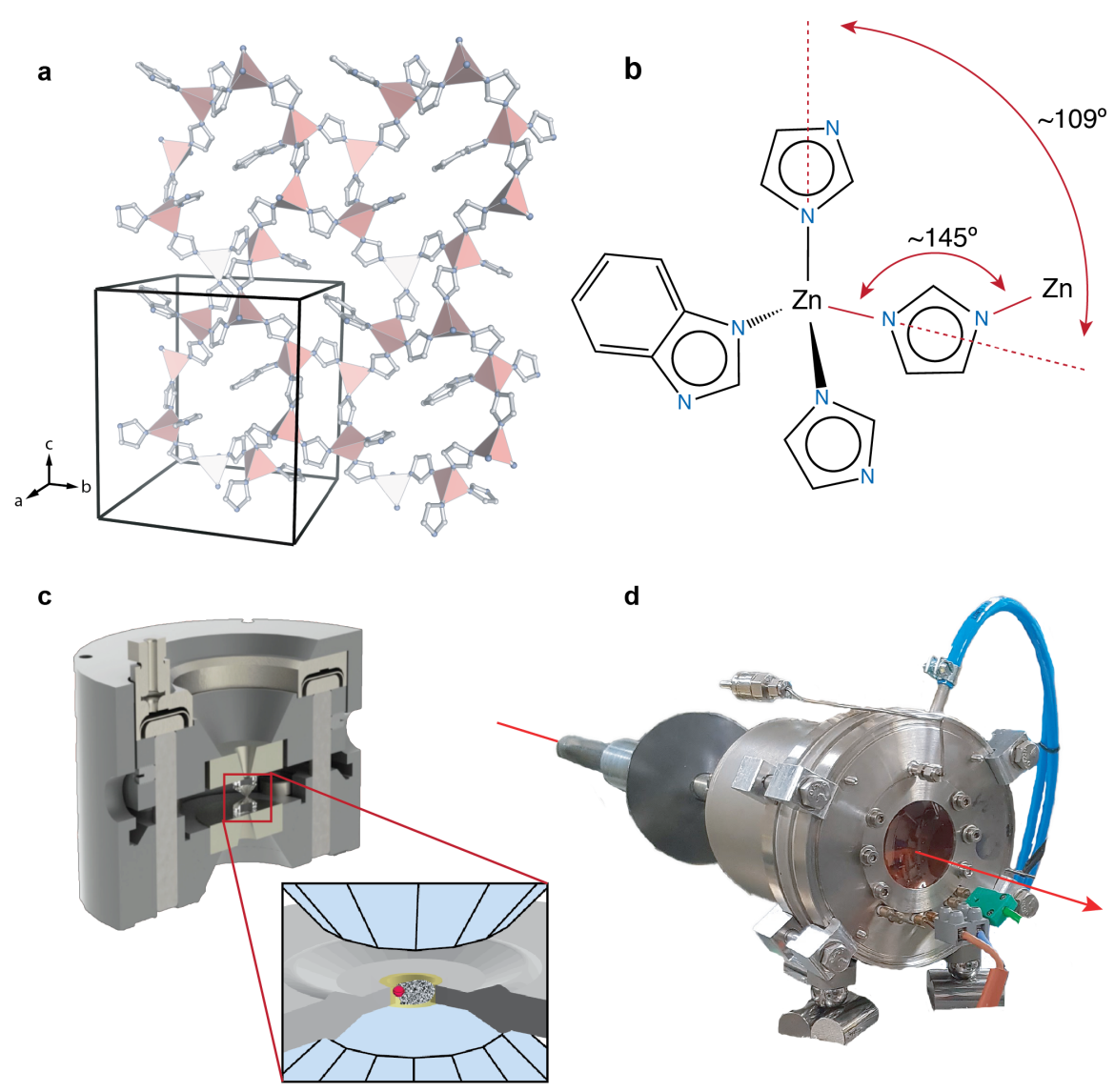

d

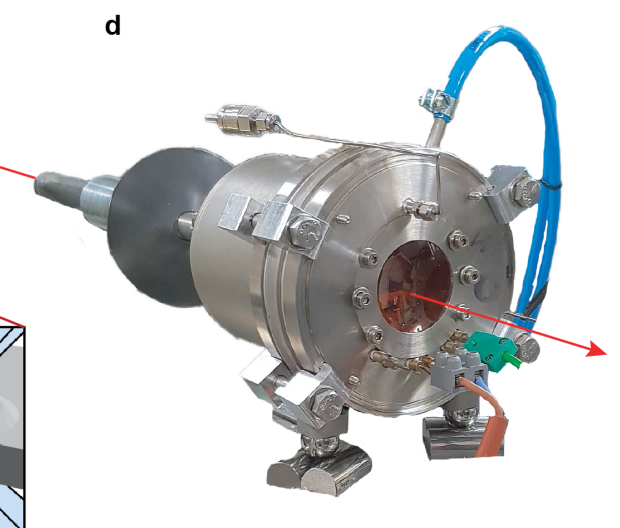

Figure 1. Crystal structure of ZIF-62 and experimental set-up

a, Representative slice of the ZIF-62 structure $\left(\mathrm{N}=\right.$ light blue, $\mathrm{C}=$ white, $\mathrm{ZnN}_{4}$ tetrahedra = pink) perpendicular to the a-axis revealing the main connectivity of 8- and 4-membered-rings. For clarity, only the range of fractional coordinates from 0.2 to 0.8 along $a$ is shown, and $\mathrm{H}$ atoms are omitted. $\mathbf{b}$, Characteristic building unit of ZIFs with four crystallographic independent linkers, including one benzimidazole for imidazole substitution, coordinating tetrahedral $\mathrm{Zn}^{2+}$ cations. Approximate bridging angles are indicated, and are similar to those of $\mathrm{SiO}_{4}$ networks of minerals. c, Schematic drawing of the membrane driven diamond anvil cell (Betsa, France) used for lab-based high- $P$ experiments. A ring-shaped membrane is inflated by gas-pressure, driving the opposing diamond anvils against each other. A gasket with a $200 \mu \mathrm{m}$ hole containing the sample material and a ruby sphere and the pressure transmitting medium is shown in the close-up view. $\mathbf{d}$, Water cooled vacuum vessel with Kapton windows and part of the X-ray collimator assembly containing an externally resistive-heated, membrane-driven DAC for simultaneous high- $P$ - $T$ powder $\mathrm{X}$-ray diffraction experiments at a synchrotron light source. The direction of beam is indicated by a red arrow. 


\section{Results and Discussion}

\section{Constructing a $P-T$ phase diagram of ZIF-62}

A resistively-heated membrane diamond anvil cell (DAC, Figure 1c and 1d) was used to investigate the phase stability of ZIF-62 in a $P-T$ range from ambient pressure to $8 \mathrm{GPa}$ and from ambient temperature to $450^{\circ} \mathrm{C}$, respectively. A crystalline sample of evacuated ZIF-62 (see methods) was loaded in the DAC together with high purity silicone oil as a non-penetrating pressure transmitting medium. In-situ characterization of the $P$ - $T$ evolution of crystalline ZIF-62 was performed with three separate synchrotron- and one lab-based X-ray diffraction experiment. The amorphization of ZIF-62 was tracked by observing the loss of diffraction peaks. Lab-based optical microscopy was performed to visually determine melting points. The resulting stability fields of crystalline, solid-amorphous, and liquid ZIF-62 are shown in Figure 2a. Solid-state amorphization and melt-formation were differentiated by morphological observations of quenched and recovered material (Figure 2b-d). 


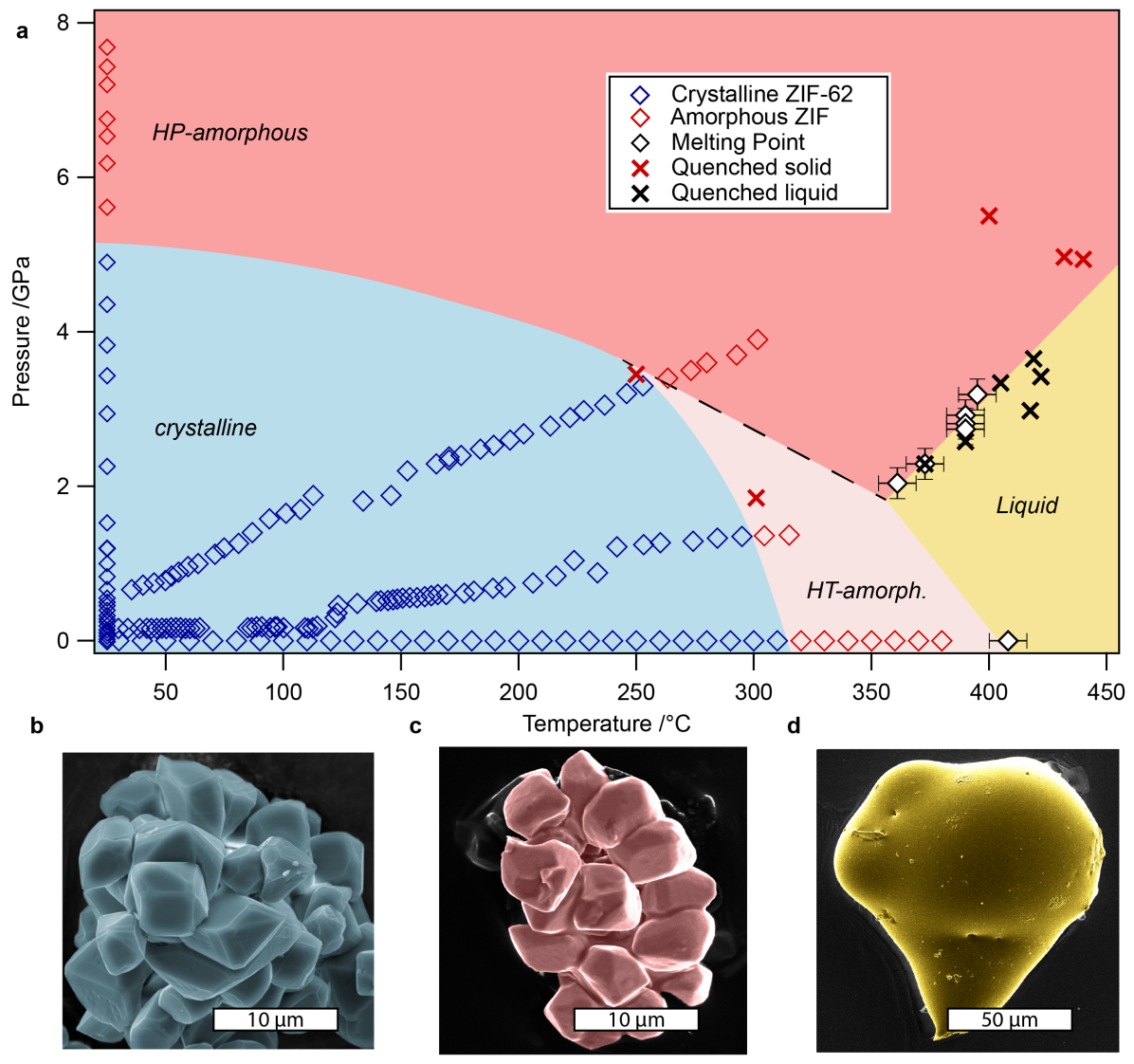

\section{Figure 2. Experimentally derived P-T phase diagram for ZIF-62}

a, The stability field of crystalline ZIF-62 is shown in blue, tracing phase analysis from PXRD (blue squares). Two distinct amorphous phases with high and low densities relative to each other are shown in dark red (high-pressure) and light red (high-temperature), respectively. This distinction is based on changing slopes of both crystalline-amorphous and solid-liquid phase-boundaries. The phase boundary between the two amorphous phases is tentatively indicated with a dashed black line.

The phase-field for liquid ZIF-62 is shown in yellow, defined by melting points (black diamonds) observed optically at high-pressure and using DSC at ambient pressure. Crosses indicate highpressure/high-temperature conditions from which the samples were quenched for morphological analysis. b, c, and d, Scanning-electron microscopic images of the three main phases of ZIF-62, in false colors corresponding to the stability fields. Crystalline, as-synthesized, ZIF-62 (blue), highpressure amorphous (red), quenched from $440{ }^{\circ} \mathrm{C}, 5 \mathrm{GPa}$ and high-pressure molten (yellow), quenched from $420^{\circ} \mathrm{C}, 3.4 \mathrm{GPa}$. 


\section{Crystalline to solid-amorphous boundary}

At ambient $P$, the ZIF-62 structure underwent $T$-induced amorphization at ca. $320^{\circ} \mathrm{C}$, which is in good agreement with a previous study ${ }^{36}$. At high- $P$ and ambient- $T$, the framework underwent amorphization at ca. $5 \mathrm{GPa}$. In comparison, a topologically identical structure containing only the unsubstituted imidazolate ligand, ZIF-4, undergoes reversible $P$-induced amorphization at ca. $1 \mathrm{GPa}^{46}$. This significant difference in high- $P$ stability at ambient- $T$ between these two ZIFs may be explained by the more sterically hindered benzimidazolate linkers present in ZIF-62 protruding into the pore cavities and offering additional stabilization against framework collapse (Figure 2a). Force-field based molecular dynamics simulations (see methods) of the elastic properties of ZIF-62 and ZIF-4 were performed to highlight this structural stabilization. The results (Supplementary Tables 1 \& 2), demonstrate higher bulk (4.7 GPa), Young's (8.7 GPa) and shear (3.6 GPa) moduli in ZIF-62, compared to ZIF-4 (2.7 GPa, 5.6 GPa and 2.4 GPa, respectively).

$P$ - and $T$-induced amorphization in ZIF-62 were found to be distinct in nature. Under pressure, the related ZIF-4 demonstrably undergoes a displacive transition since the material was shown to revert to a crystalline phase upon decompression ${ }^{46}$. We have similar evidence for short-range ordering in ZIF-62 from in-situ Raman spectroscopic data. The loss of the characteristic phonon frequencies and the internal modes was found to be fully reversed upon decompression (Supplementary Figure 1). A synchrotron high-pressure PXRD experiment was carried out on ZIF-62, and demonstrated amorphization to occur at $5 \mathrm{GPa}$. This amorphous phase reverts to the starting crystalline phase upon decompression, and thus is assigned as a displacive transition (Fig. 2a, Supplementary Figure 2). This is consistent with a displacive 
mechanism of formation. In contrast, thermal amorphization of ZIF-62 is irreversible and is ascribed to a reconstructive phase transition. Diffraction patterns from samples of ZIF-62 amorphized at (i) $7.6 \mathrm{GPa}$ and $25^{\circ} \mathrm{C}$, and (ii) $350{ }^{\circ} \mathrm{C}$ under vacuum, show complete loss of all diffraction peaks in each case (Supplementary Figure 3).

Amorphization at simultaneous high- $P$ and $-T$ is therefore expected to share aspects of both processes. Moreover, the changing gradient of the crystalline to solidamorphous transition (Figure 2a) found across $P$ - $T$-space is indicative of high- $P$ and high- $T$ amorphous phases with different densities. This can be derived from the $\Delta S / \Delta V$ term of the Clausius-Clapeyron relation for a boundary with a changing gradient as opposed to having a constant gradient.

We denote the high- $P$ and high- $T$ amorphous phases $a_{\mathrm{HP}}$ and $a_{\mathrm{HT}}$, and the corresponding crystalline-amorphous transitions $c-a_{\mathrm{HP}}$ and $c-a_{\mathrm{HT}}$, respectively. The difference in slopes of the transitions dominated by either $\mathrm{c}-a_{\mathrm{HP}}$ or $\mathrm{c}-a_{\mathrm{HT}}$ in $P$ - $T$-space implies that $\Delta V_{c-a H T}<\Delta V_{c-a H P}$ and consequently, we find that $a_{H P}$ is of higher density than $a_{H T}$, as anticipated under the influence of pressure. We defined an approximate boundary between $a_{\mathrm{HT}}$ and $a_{\mathrm{HP}}$ by extrapolating the $\mathrm{c}-a_{\mathrm{HP}}$ boundary to higher pressures (dashed line in Figure 2a).

\section{Solid-amorphous to liquid boundary}

The melting point of ZIF-62 at ambient- $P$ was identified at ca. $430{ }^{\circ} \mathrm{C}$ by differential scanning calorimetry (DSC) measurements (Supplementary Figure 4). Melting points at high- $P$ ranging from approximately $2 \mathrm{GPa}$ to $3.5 \mathrm{GPa}$ were determined visually and are shown in Figure 2a. A representative video showing the melting is shown in 
Supplementary Video 1. Six independent melting points at variable $P$ were fitted linearly with a slope of $d P / d T=0.031(4)$. Achievable $P-T$ conditions in DAC experiments are constrained to lie on isochores dictated by the properties of the pressure-transmitting medium. For this reason, it was not technically feasible to target $P$ - $T$ conditions for intermediate- $P$ and high- $T$. However, we are able to constrain the upper limit of the melting curve up to approximately $5 \mathrm{GPa}$ by solids that were quenched from high- $P-T$ conditions indicated in Figure $2 a$ by red crosses, showing no morphological signs of melting (Figure 2c).

The negative slope of the melting curve interpolated from ambient pressure to $<2$ GPa (boundary between pale red and yellow in Figure 2a) implies a solid phase with lower density than the liquid phase. At pressures $>2 \mathrm{GPa}$, we find a melting curve with a positive gradient (boundary between dark red and yellow in Figure 2a), implying the opposite, a solid phase with a higher density than the liquid phase. Both conditions are in accordance with, and support, our model of two distinct amorphous phases $a_{\mathrm{HT}}$ and $a_{\mathrm{HP}}$. The intersection of the low- and high- $P$ melting curve with the assumed boundary between $a_{\mathrm{HT}}$ and $a_{\mathrm{HP}}$ (dashed line in Figure 2a) defines a triple point.

\section{Structural evolution}

$T$ - and $P$-dependent lattice parameters of crystalline ZIF-62 were refined from synchrotron and lab-based X-ray powder diffraction data. Figure 3a displays the unit cell volume of ZIF-62 in P-T-space. Pure $T$ - and $P$-dependent unit cell dimensions are shown in Figure 3 and Supplementary Figure 5, respectively. Experimentally derived bulk moduli and linear thermal expansion coefficients are given in 
Supplementary Tables $3 \& 4$. Following quasi-linear thermal volume expansion upon heating from ambient- $T$ up to $270^{\circ} \mathrm{C}$, a significant volume collapse is observed between $270{ }^{\circ} \mathrm{C}$ and complete amorphization at $320^{\circ} \mathrm{C}$. Thermal expansion here is, however, not negative, as shown in Figure $3 b-$ consistent with previous computational work indicating positive thermal expansion for ZIFs of cag topology ${ }^{47}$. This collapse of the unit cell volume coincides with a decrease in diffraction intensity and is due to progressive collapse to a higher density phase. Upon hydrostatic compression at ambient temperature, the $c$-axis is the least compressible direction. Under vacuum, thermal expansion is net-zero along the $c$-axis, slightly negative along the a-axis, and strongly positive along the $b$-axis. This extreme anisotropic behavior can be understood from the topology of the rings formed by interconnected $\mathrm{Zn}$ sites. A network of 8-membered rings extends perpendicular to a with blm linkers protruding along the major-axes of their elliptical shape. This direction is dominantly parallel to $b$. Shearing of the rings perpendicular to the blm linkers by expansion along $c$ is therefore sterically blocked. A network of 6-membered rings extending predominantly perpendicular to $b$ is of a more isotropic and rigid geometry, resulting in a subtler response along $a$ and $c$ compared to $b$ (Supplementary Figure 6$)$. In comparison, structurally identical but unsubstituted ZIF-4 shows no hindrance by bulky benzimidazole linkers and indeed strongly contracts along $c$ while also expanding along $b$ (Supplementary Figure $6 \& 7$ ).

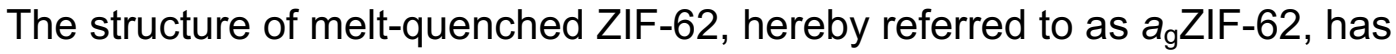
previously been investigated by X-ray total scattering ${ }^{36}$. It was found that the $\mathrm{Zn}-\mathrm{N}$ association is virtually identical between crystalline precursor and melt-quenched glass. Permanently porous glasses are rare. Where they do exist, the porosity in these materials is typically generated by post-synthetic chemical or physical 
treatment. Conversely, we have recently demonstrated that glasses derived from MOFs can be made permanently accessible to incoming guest molecules such as $\mathrm{CO}_{2}$ and $\mathrm{CH}_{4}{ }^{40}$. Gas adsorption isotherms were used to identify and characterise the accessible pores of crystalline ZIF-62 and $a_{\mathrm{g}}$ ZIF-62, by quenching from $T_{\mathrm{m}}$ at ambient pressure. Crystalline ZIF-62 takes up $\mathrm{H}_{2}$ at $77 \mathrm{~K}$ and $\mathrm{CO}_{2}$ at $273 \mathrm{~K}$ (Supplementary Figure 8). However, at $77 \mathrm{~K}$, diffusion limitations prevent the ingress of $\mathrm{N}_{2}$ guest molecules ${ }^{36}$. $\mathrm{CH}_{4}$ measurements were also performed to show that this is not a size exclusion effect (Supplementary Figure 8).

The permanent porosity of $a_{\mathrm{g}} \mathrm{ZIF}-62$ was confirmed by recording its uptake of $\mathrm{CO}_{2}$ at $273 \mathrm{~K}$ (Figure 3c, Table 1). This material reversibly adsorbs $20.1 \mathrm{~mL}(\mathrm{STP}) / \mathrm{g}$ at a pressure of one bar, which equates to $3.8 \mathrm{wt} \%$. In contrast to the crystalline material,

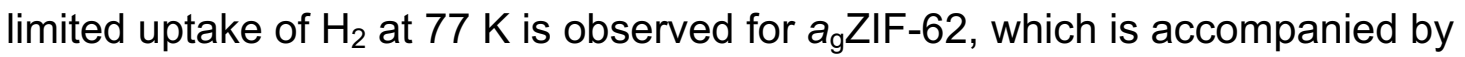
significant hysteresis (Supplementary Figure 9). This hysteresis is ascribed to diffusion limitations at this temperature, on the basis of the reversibility of the $\mathrm{H}_{2}$ isotherm measured at $195 \mathrm{~K}$ and the $\mathrm{CO}_{2}$ isotherm measured at $273 \mathrm{~K}$. At $77 \mathrm{~K}$, a small component of $\mathrm{H}_{2}$ cannot be removed in the desorption branch of $\mathrm{ag}_{\mathrm{g}} \mathrm{ZIF}-62$ at very low pressures under practical operating conditions. To confirm that this does not correspond to irreversible chemical modification of $a_{g} Z I F-62$, the residual gas was removed, by warming the sample to room temperature under vacuum. It thus appears that $a_{g}$ ZIF-62 kinetically traps a small amount of $\mathrm{H}_{2}$, as has been observed in some crystalline systems, ${ }^{48}$ where the some of the pore openings and $\mathrm{H}_{2}$ have similar diameters. 
Table 1. Gas uptakes measured by adsorption isotherms and compared with calculated values. Uptakes are given in units of $\mathrm{mL}(\mathrm{STP}) / \mathrm{g}$ at a pressure of one bar.

\begin{tabular}{ccccc}
\hline Gas (Kinetic Diameter / A) & $\mathrm{H}_{2}(\mathbf{2 . 9})$ & $\mathrm{CO}_{2}(\mathbf{3 . 3})$ & $\mathrm{O}_{2}(\mathbf{3 . 4 6})$ & $\mathbf{N}_{\mathbf{2}}$ (3.64) \\
Temperature / K & 77 & 273 & 273 & 77 \\
\hline $\mathrm{ZIF-62}$ & 130 & 39 & - & 0 \\
$a_{\mathrm{g}} \mathrm{ZIF-62}$ & 9.3 & 20.1 & 1.5 & 0 \\
\hline
\end{tabular}

With regards to ZIF-4, first principle molecular dynamics calculations combined with neutron total scattering data ${ }^{37}$ and positron annihilation lifetime spectroscopy ${ }^{49}$ have also shown that both the liquid and the quenched glass retain porous characteristics similar to the crystalline phase. Here, ex-situ Raman spectra of high- $P$ meltquenched ZIF-62 (Supplementary Figure 10) are very similar to those of the crystalline starting material, with all Raman modes associated both with the phonon frequencies $\left(<300 \mathrm{~cm}^{-1}\right)$ and the internal modes of organic linkers $\left(>300 \mathrm{~cm}^{-1}\right)$ observed. The only significant difference between the crystalline and the meltquenched material is the smoothing of the continuum at wavenumbers $<300 \mathrm{~cm}^{-1}$. 
a

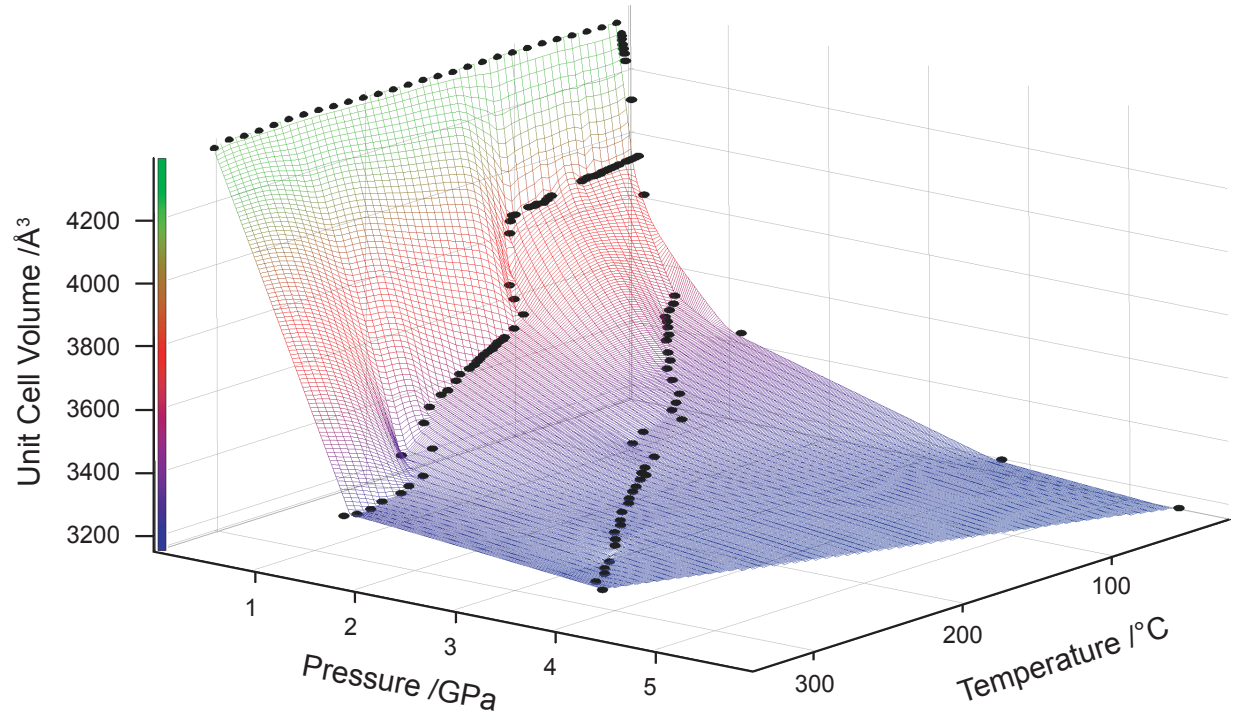

b
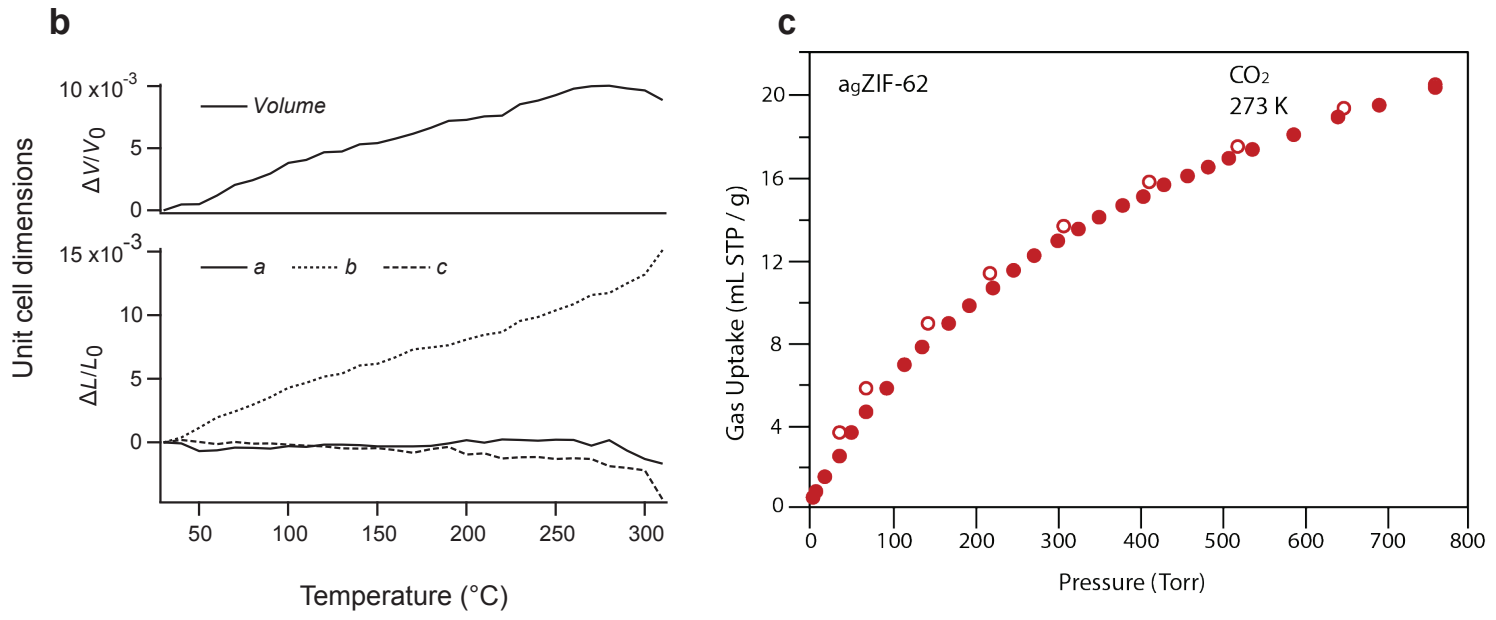

Figure 3. Structural evolution of ZIF-62 in P-T-space

a, Unit cell volume of crystalline ZIF-62 as a function of $P$ and $T$ from Rietveld refinements. A graphically interpolated surface is included as a guide to the eye. $\mathbf{b}$, Thermal expansion under vacuum, indiscernible on the scale of hydrostatic compression, plotted as relative and normalized change. Strong anisotropic expansion is apparent from the individual crystallographic axes. A volume collapse precedes complete amorphization above $270{ }^{\circ} \mathrm{C} . \mathbf{c}, \mathrm{CO}_{2}$ gas adsorption isotherm for the ambient pressure melt-quenched ZIF-62 glass. Filled symbols represent adsorption, open symbols represent desorption. 
A second, high- $P-T$ synchrotron experiment was carried out using ZIF-4

(Supplementary Figure 11). At ambient $T$, amorphization, recrystallization to the dense ZIF-zni phase and melting were confirmed at ca $250^{\circ} \mathrm{C}, 320^{\circ} \mathrm{C}$ and $580^{\circ} \mathrm{C}$ as expected ${ }^{38}$. Application of pressure however resulted in a dramatic lowering of amorphization and recrystallization, to $<200^{\circ} \mathrm{C}$ and ca. $250^{\circ} \mathrm{C}$, and melting was observed at high pressures below the ambient melting point $T_{\mathrm{m}}$. This hints at the transferability in the pressure-induced lower temperature melting phenomenon.

\section{Molecular simulations}

In order to give microscopic and thermodynamic insight into the evolution of the ZIF melting processes upon application of pressure, we performed first principles molecular dynamics (FPMD) simulations based on a quantum chemical description of the system at the Density Functional Theory (DFT) level, detailed in the Supplementary Methods. These simulations were performed on both ZIF-4 and ZIF62 , in the $0-5 \mathrm{GPa}$ pressure range at the previously identified computational melting point (for ZIF-4) of $1200 \mathrm{~K}^{37}$. Given the limited timescale attainable through FPMD simulations, these simulations probe the melting behavior of the crystalline phase near the experimental crystalline to high- $T$-amorphous and high- $T$-amorphous to liquid phase transitions.

Analysis of the FPMD simulations suggests that the mechanism behind the melting of the ZIFs is not significantly affected by pressure. On the other hand, the application of pressure in the GPa range affects both the dynamics and thermodynamics of the systems. Figure 4a depicts the potential of mean force (PMF) 
for the zinc-nitrogen coordination, calculated from the free FPMD simulations of ZIF62 at various pressures. There is a clear decrease in the free energy barrier upon increasing pressure from $0.1 \mathrm{GPa}$ to $5 \mathrm{GPa}$, associated with the rupture of the coordination bond (Table 2, Supplementary Table 5). This can be linked to the softening of the Im-Zn-Im angle upon pressurization, which was previously shown to be the driving force of the pressure-induced amorphization of ZIF-8 and ZIF-4 at room temperature ${ }^{47,50}$. Confirmation of this behaviour is also found in the dynamics of the $\mathrm{Zn}-\mathrm{N}$ coordination bonds, for which the average time between $\mathrm{Zn}-\mathrm{N}$ cleavage events during the FPMD is shown in Figure 4b. This softening of the porous framework's coordination upon pressurization means that melting becomes thermodynamically easier upon application of pressure, explaining the observed negative slope of the ZIF-62 melting curve in $P$ - $T$-space. Simulations were also carried out using the published structural model for ZIF-4, finding similar results (Supplementary Figure 12) and reinforcing the idea that the pressure-induced lowering of the melting point can be extended to other ZIFs - similarly to the pressure-induced amorphization mechanism ${ }^{50}$.

This is a very counter-intuitive behaviour, since for most solids application of pressure inhibits melting. This raises the possibility that the liquid-solid phase relations of amorphous ZIFs share similarities with those of other disordered framework materials such as $\mathrm{H}_{2} \mathrm{O}$ ice. Negative melting curves have also been inferred from separate ambient $T$, and ambient $P$ experiments on inorganic zeolites ${ }^{51}$, though importantly these deal with collapse of the crystalline state to amorphous solid states, and not to macroscale liquids, as in this study. 
There is a large difference in chemistry between ZIFs and zeolites, and specifically the weaker $\mathrm{Zn}-\mathrm{N}$ bonding compared to $\mathrm{Si}-\mathrm{O}$ interactions ${ }^{52}$. This facilitates melting to a liquid at accessible temperatures and allows us to perform the high- $P-T$ experiments required to confirm the negative melting behaviour in these materials. In contrast, the $\mathrm{Zn}-\mathrm{N}$ bond is stronger than the interactions present in organic ionic liquids ${ }^{53}$, and results not only in higher melting points of ZIFs, but also in a different mechanism of melting. For example, whilst melting in examples of ionic liquids has been shown to result in the complete conformational freedom of component species ${ }^{54}$, and molten salt structures such as $\mathrm{ZnCl}_{2}$ have been shown to retain tetrahedral, or octahedral coordination ${ }^{55}$, the melting of a ZIF does not result in fully undercoordinated $\mathrm{Zn}^{2+}$ centres or $\mathrm{Zn}(\mathrm{Im})_{4}$ fragments ${ }^{37}$, but in a strongly associated liquid — once again, similar to liquid water.

Table 2. Evolution of the $\mathrm{Zn}-\mathrm{N}$ coordination free energy barrier with pressure, for ZIF-62

\begin{tabular}{cccccc}
\hline Pressure (GPa) & 0.5 & 1.0 & 2.0 & 4.0 & 5.0 \\
$\Delta G^{\ddagger}(\mathrm{kJ} / \mathrm{mol})$ & 50 & 45 & 45 & 37 & 34 \\
\hline
\end{tabular}



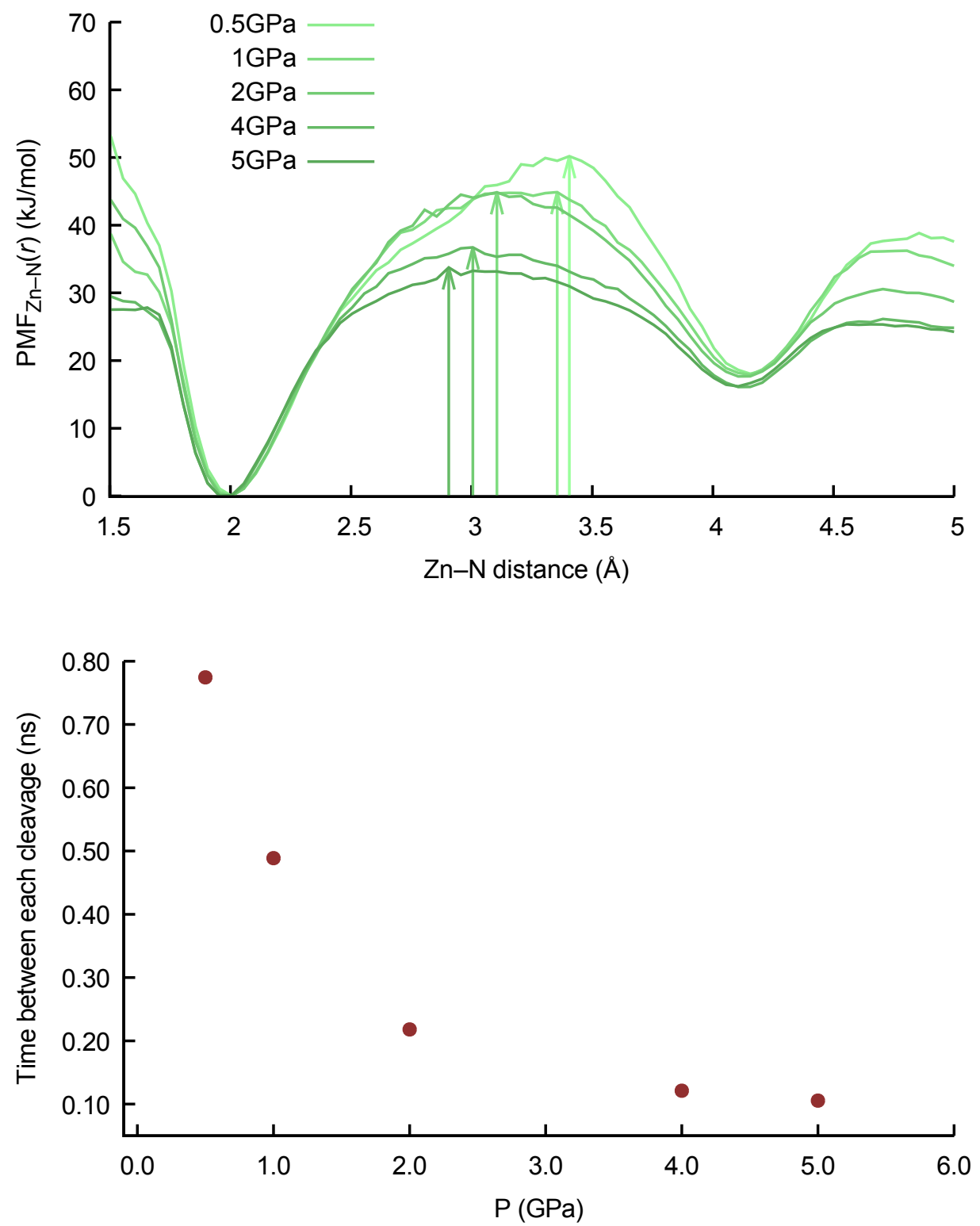

Figure 4. Potential of mean force for the $\mathrm{Zn}-\mathrm{N}$ distance, and dynamics of $\mathrm{Zn}-\mathrm{N}$ cleavages.

a. Potential of mean force for the $\mathrm{Zn}-\mathrm{N}$ distance, obtained from first-principles molecular dynamics of ZIF-62 at pressures between $0.1 \mathrm{GPa}$ and $5 \mathrm{GPa}$, at a temperature of $1,200 \mathrm{~K}$. The values of the free energy barrier $\Delta G^{\neq}$corresponding to the breaking of the $\mathrm{Zn}-\mathrm{N}$ coordination bond (visible as vertical arrows) are given in Table 2.

b. Average time between events of cleavage of a given $\mathrm{Zn}-\mathrm{N}$ bond, averaged from the firstprinciples MD trajectories, as a function of pressure applied. 


\section{Conclusion}

The $P$-T phase diagram of the metal-organic frameworks ZIF-4 and ZIF-62 have been created by combining in-situ X-ray diffraction and optical microscopy with thermodynamic considerations. Importantly, we show how the stability field of the liquid phase extends substantially towards lower temperatures at increasing hydrostatic pressure. Aside from the fundamental interest in negative Clapeyron slope melting behaviour displayed here, reducing the melting temperature is an important objective in developing new MOF glasses. We have also demonstrated the permanent accessible porosity of $a_{g} Z \mathrm{IF}-62$, which will encourage others to research porous glasses, as compounds decompose at temperatures below their potential melting temperatures ${ }^{37}$. The possibility to synthesize liquid MOF states via highpressure thermal melting opens a route to synthesis of a very wide range of amorphous MOFs. The pressures regime required for lowering the melting temperatures is achievable through industrially viable processes such as hotpressing and specialised autoclaves.

The exploration of $P$ - $T$-space here also provides essential information on the stability of individual phases and the nature of transformations between those phases. The density contrast delineated between high- $P$ and high- $T$ amorphous MOF phases confirms distinct amorphization mechanisms and infers the existence of point of coexistence between these two amorphous phases and the crystalline state. For example, pressure- or temperature- induced transitions have been found in non-ZIF metal-organic frameworks such as $\mathrm{PCN}-250^{56}$ and $[\mathrm{NH} 4]\left[\mathrm{Zn}(\mathrm{HCOO})_{3}\right]^{57}$. This already hints at the possibility for rich phase diagrams of the MOF family, even at relatively low temperatures and pressures. 
Finally, the study of MOFs under simultaneous high- $T$ and $-P$ ultimately links polymer chemistry with mineralogy. Tools which are used for non-ambient studies in mineral sciences have been adapted for the MOF field, providing a compelling new perspective on MOFs and drawing parallels between their rich phase transition behavior, and that of classical inorganic solids such as $\mathrm{SiO}_{2}$. 


\section{Methods}

ZIF-62 and ZIF-4 were prepared and desolvated according to a procedure adapted from the literature ${ }^{58}$. Specifically, ZIF-62 and ZIF-4 were synthesized by a solvothermal method in which $1.515 \mathrm{~g} \mathrm{Zn}\left(\mathrm{NO}_{3}\right)_{2} \cdot 6 \mathrm{H}_{2} \mathrm{O}(8 \mathrm{mmol}), 7.35 \mathrm{~g}$ imidazole (108 mmol), and $1.418 \mathrm{~g}$ benzimidazole (12 mmol) (ZIF-62), and $1.2 \mathrm{~g} \mathrm{Zn}\left(\mathrm{NO}_{3}\right)_{2} \bullet$ $6 \mathrm{H}_{2} \mathrm{O}(4.03 \mathrm{mmol})$, and $0.9 \mathrm{~g}$ imidazole (13.2 mmol) (ZIF-4) were dissolved in $75 \mathrm{ml}$ of dimethylformamide (DMF) and transferred into a $100 \mathrm{ml}$ glass jar. The jars were sealed tightly and heated to $130{ }^{\circ} \mathrm{C}$ for $48 \mathrm{~h}$ in an oven. The filtered off, assynthesized ZIFs were soaked in dichloromethane for solvent exchange during 24 hours and subsequently desolvated in a vacuum oven at $0.01 \mathrm{mbar}$ and $140{ }^{\circ} \mathrm{C}$. A sample of $a_{\mathrm{g}}$ ZIF-62 was prepared by heating ZIF-62 under argon in a tube furnace to $430^{\circ} \mathrm{C}$, and cooling naturally.

The resulting compositions are $\mathrm{Zn}(\mathrm{Im})_{1.76}(\mathrm{blm})_{0.24}$ for ZIF-62, and $\mathrm{Zn}(\mathrm{Im})_{2}$ for ZIF-4 as determined by high-performance liquid chromatography (HPLC) (Supplementary Methods, Supplementary Figure 13). The XRD patterns and Raman spectra of the starting materials are shown in Supplementary Figure 14.

Ambient pressure variable temperature powder X-ray diffraction data were collected under vacuum on a Bruker D8 Advance diffractometer equipped with a MRI radiation-heating stage. Pure silicon powder was mixed with ZIF-62 and ZIF-4 and used as an internal standard for sample displacement corrections.

Combined high- $P$ and high-T powder diffraction data of ZIF-62 and ZIF-4 were obtained at beamline 115, Diamond Light Source, Didcot (UK) (Supplementary Figures 15 - 19). A membrane-driven diamond anvil cell (DAC) was equipped with an external coiled resistive heater. The DAC was contained in a water-cooled vacuum vessel with Kapton windows. High-purity silicone oil (AP100, Sigma-Aldrich) 
was used as pressure transmitting medium. Data were collected at a wavelength of $\lambda$ $=0.4246 \AA$ using a MAR345 detector. Diffraction images were integrated using the DAWN software suite ${ }^{59}$. Internal pressures were calculated from the unit cell volume of $\mathrm{NaCl}$ using equation-of-state parameters from literature ${ }^{60}$. A K-type thermocouple with an accuracy of $<0.4 \%$ was glued close to one of the diamonds to provide temperature measurements. Before each heating ramp, the DAC-internal $P$ was raised to a target value by inflating the membrane. This membrane pressure was kept constant throughout the individual runs. $T$ was raised continuously at an average rate of $0.5{ }^{\circ} \mathrm{C} / \mathrm{min}$ for run 1 and $1{ }^{\circ} \mathrm{C} / \mathrm{min}$ for run 2 , resulting in simultaneous increase of $T$ and $P$. Diffraction patterns were collected in approximately $10^{\circ} \mathrm{C}$ temperature increments.

Melting points of ZIF-62 at high- $P$ were determined visually from lab-based, externally heated DAC experiments in silicone oil as pressure transmitting medium (Supplementary Figures 20 \& 21). Optical images of the recovered ZIF-4 samples are in Supplementary Figure 22. The temperature was measured using a K-type thermocouple glued to one of the diamonds. $P$ was determined from the shift of the fluorescence peaks of ruby ${ }^{61}$ corrected for the $T$-induced shift ${ }^{62} . T$ was increased continuously at approximately $5{ }^{\circ} \mathrm{C} / \mathrm{min}$, while initial membrane pressure was kept constant. The lowest possible pressure at a certain temperature is dictated by the isochoric path followed through $T$ increase. Therefore, it was not possible to access pressures below $2 \mathrm{GPa}$ between $300{ }^{\circ} \mathrm{C}$ and $350{ }^{\circ} \mathrm{C}$. After reaching maximum $T$ and $P$ conditions, the external heater was switched off resulting in an approximate cooling rate of $2.5 \mathrm{~K} / \mathrm{min}$. $P$ was released after reaching room $T$. The gaskets containing samples were transferred to SEM stubs, kept under vacuum for $48 \mathrm{~h}$ to evaporate silicone oil residues, and carbon coated for subsequent SEM imaging. 
Back-scattered electron images were acquired on a FEI Qemscan Quanta650F operated at $10 \mathrm{kV}$ at a working distance of $10 \mathrm{~mm}$.

Rietveld refinement of experimental diffraction patterns was performed using TOPAS-Academic $v 6^{63}$ based on a ZIF-62 and ZIF-4 structure model from literature ${ }^{26}$. Synchrotron based powder XRD data was fitted sequentially using experimental peak shapes from $\mathrm{LaB}_{6}$ reference measurements. An experimental, fixed background profile measured from an empty DAC in the vacuum vessel was fitted together with $5^{\text {th }}$ order Chebychev polynomial. $8^{\text {th }}$ order spherical harmonics were applied to intensities for preferred orientation correction. Lattice parameters, scale factors, and lorentzian strain was refined individually for all measurements. Numeric fit results are presented in the supporting information. Lab-based, variable-temperature PXRD data sets were fitted parametrically. An internal silicon standard allowed for refining individual sample displacements using fixed, temperature dependent standard lattice parameters for Si at each temperature. Subsequently, ZIF-62 and ZIF-4 were fitted using fixed, previously refined, specimen displacements.

Gas adsorption isotherms were measured by a volumetric method using ultra-high purity gases. Prior to analysis, the samples were degassed under a dynamic vacuum at $10^{-6}$ Torr for $10-20$ hours at $130-250^{\circ} \mathrm{C}$. Accurate sample masses were calculated using degassed samples after sample tubes were backfilled with nitrogen. 


\section{Acknowledgements}

R.N.W. acknowledges support of the EPSRC in the form of a DTG Graduate Studentship. R.N.W. and A.M.B thank Prof. Chris Hunter and his group, at the Department of Chemistry (University of Cambridge), for the use of their HPLC facilities. T.D.B. thanks the Royal Society for a University Research Fellowship and for their support (UF150021). C.Z. acknowledges the financial support of the Elite Research Travel Scholarship from the Danish Ministry of Higher Education and Science. We thank Diamond Light Source for access to beamline I15 (EE16133 and EE19046-1). This work benefitted from the financial support of ANRT (these CIFRE 2015/0268) and access to HPC platforms provided by a GENCl grant (A0030807069). Gas sorption on the $a_{g}$ ZIF-62 was supported by a grant from the National Science Foundation, Division of Chemistry under award number CHE1661655. Laurine Friche, Nisansala Bandata and Omid T. Qasvini (Massey Unviersity) are thanked for technical assistance, alongside Dr. Brian R. Pimentel (UCSD). British Crown Owned Copyright 2018/AWE. Published with permission of the Controller of Her Britannic Majesty's Stationary Office.

\section{Contributions}

T.D.B. and S.A.T.R. designed the project. R.N.W., G.I.L, S.A., S.G.M, A.K.K., D.D., M.T.W., S.F., C.Z., C. W and T.D.B performed the X-ray powder diffraction experiments. S.G.M. designed and constructed the high-P-T PXRD equipment, R.N.W. performed melting point determinations, Raman spectroscopy and microscopy, and analysed XRD and spectroscopic data. H.P. and C.Z. performed DSC measurements. A.M.B performed HPLC measurements. X.Y, S.M.C (University of California San Diego) and S.G.T performed gas sorption measurements. R.G. and 
F.-X.C. designed, performed, and analysed the molecular simulations. All Authors participated in discussing the data. R.N.W. and T.D.B. wrote the manuscript with input from all authors.

\section{Data Availability}

The data that support the findings of this study are available from the corresponding author on request.

\section{Additional information}

Supplementary Information is available in the online version of the paper.

Correspondence and requests for materials should be addressed to T.D.B.

\section{Competing interests}

The authors declare no competing financial interests. 


\section{References}

1. Moghadam, P. Z. et al. Development of a Cambridge Structural Database Subset: A Collection of Metal-Organic Frameworks for Past, Present, and Future. Chem. Mater. 29, 2618-2625 (2017).

2. Kim, H. et al. Water harvesting from air with metal-organic frameworks powered by natural sunlight. Science 356, 430-434 (2017).

3. Mason, J. A. et al. Methane storage in flexible metal-organic frameworks with intrinsic thermal management. Nature 527, 357 (2015).

4. Yoon, J. W. et al. Selective nitrogen capture by porous hybrid materials containing accessible transition metal ion sites. Nat. Mater. 16, 526 (2016).

5. Denny Jr, M. S., Moreton, J. C., Benz, L. \& Cohen, S. M. Metal-organic frameworks for membrane-based separations. Nat. Rev. Mater. 1, 16078 (2016).

6. Mondloch, J. E. et al. Destruction of chemical warfare agents using metalorganic frameworks. Nat. Mater. 14, 512 (2015).

7. Horike, S., Shimomura, S. \& Kitagawa, S. Soft porous crystals. Nat Chem 1, 695-704 (2009).

8. Dissegna, S., Epp, K., Heinz, W. R., Kieslich, G. \& Fischer, R. A. Defective Metal-Organic Frameworks. Adv. Mater. (2018). doi:10.1002/adma.201704501

9. Schneemann, A. et al. Flexible metal-organic frameworks. Chem. Soc. Rev. 43, 6062-6096 (2014).

10. Bennett, T. D., Cheetham, A. K., Fuchs, A. H. \& Coudert, F.-X. Interplay between defects, disorder and flexibility in metal-organic frameworks. Nat Chem 9, 11-16 (2017).

11. Slater, A. G. \& Cooper, A. I. Function-led design of new porous materials. 
Science (80-. ). 348, aaa8075 (2015).

12. Kitagawa, S. Future Porous Materials. Acc. Chem. Res. 50, 514-516 (2017).

13. Coudert, F.-X. Responsive Metal-Organic Frameworks and Framework Materials: Under Pressure, Taking the Heat, in the Spotlight, with Friends. Chem. Mater. 27, 1905-1916 (2015).

14. Krause, S. et al. A pressure-amplifying framework material with negative gas adsorption transitions. Nature 532, 348 (2016).

15. Carrington, E. J. et al. Solvent-switchable continuous-breathing behaviour in a diamondoid metal-organic framework and its influence on $\mathrm{CO} 2$ versus $\mathrm{CH} 4$ selectivity. Nat. Chem. 9, 882 (2017).

16. Longley, L., Li, N., Wei, F. \& Bennett, T. D. Uncovering a reconstructive solidsolid phase transition in a metal-organic framework. R. Soc. Open Sci. 4, (2017).

17. Lapidus, S. H., Halder, G. J., Chupas, P. J. \& Chapman, K. W. Exploiting High Pressures to Generate Porosity, Polymorphism, And Lattice Expansion in the Nonporous Molecular Framework Zn(CN)2. J. Am. Chem. Soc. 135, 76217628 (2013).

18. Robert M. Hazen, R. T. D. High-temperature and High-pressure Crystal Chemistry. Reviews in Mineralogy and Geochemistry 41, (The Mineralogical Society of America, 2003).

19. Hurlbut, C. S. \& Klein, C. Manual of Mineralogy (After Dana). (John Wiley \& Sons, 1977).

20. Dove, M. T. et al. Crystal structure of the high-pressure monoclinic phase-II of cristobalite, SiO2. Mineral. Mag. 64, 569-576 (2000).

21. Hemley, R. J., Jephcoat, A. P., Mao, H. K., Ming, L. C. \& Manghnani, M. H. 
Pressure-induced amorphization of crystalline silica. Nature 334, 52 (1988).

22. Natarajan, S. \& Mahata, P. Metal-organic framework structures - how closely are they related to classical inorganic structures? Chem. Soc. Rev. 38, 23042318 (2009).

23. Decurtins, S., Schmalle, H. W., Schneuwly, P., Ensling, J. \& Guetlich, P. A concept for the synthesis of 3-dimensional homo-and bimetallic oxalatebridged networks [M2 (ox) 3] n. Structural, Moessbauer, and magnetic studies in the field of molecular-based magnets. J. Am. Chem. Soc. 116, 9521-9528 (1994).

24. Sadakiyo, M., Yamada, T., Honda, K., Matsui, H. \& Kitagawa, H. Control of Crystalline Proton-Conducting Pathways by Water-Induced Transformations of Hydrogen-Bonding Networks in a Metal-Organic Framework. J. Am. Chem. Soc. 136, 7701-7707 (2014).

25. Huskić, I., Pekov, I. V, Krivovichev, S. V \& Friščić, T. Minerals with metalorganic framework structures. Sci. Adv. 2, (2016).

26. Banerjee, R. et al. High-throughput synthesis of zeolitic imidazolate frameworks and application to CO2 capture. Science 319, 939-943 (2008).

27. Park, K. S. et al. Exceptional chemical and thermal stability of zeolitic imidazolate frameworks. Proc. Natl. Acad. Sci. 103, 10186-10191 (2006).

28. McKellar, S. C. \& Moggach, S. A. Structural studies of metal-organic frameworks under high pressure. Acta Crystallogr. Sect. B 71, 587-607 (2015).

29. Cruciani, G. Zeolites upon heating: Factors governing their thermal stability and structural changes. J. Phys. Chem. Solids 67, 1973-1994 (2006).

30. Hazen, R. M. Zeolite Molecular Sieve 4A: Anomalous Compressibility and 
Volume Discontinuities at High Pressure. Science 219, 1065-7 (1983).

31. Su, Z., Miao, Y.-R., Zhang, G., Miller, J. T. \& Suslick, K. S. Bond breakage under pressure in a metal organic framework. Chem. Sci. 8, 8004-8011 (2017).

32. Chapman, K. W., Sava, D. F., Halder, G. J., Chupas, P. J. \& Nenoff, T. M. Trapping Guests within a Nanoporous Metal-Organic Framework through Pressure-Induced Amorphization. J. Am. Chem. Soc. 133, 18583-18585 (2011).

33. Zhao, P. et al. Pressure-induced oversaturation and phase transition in zeolitic imidazolate frameworks with remarkable mechanical stability. Dalt. Trans. 44, 4498-4503 (2015).

34. Henke, S. et al. Pore closure in zeolitic imidazolate frameworks under mechanical pressure. Chem. Sci. (2018). doi:10.1039/C7SC04952H

35. Bennett, T. D. et al. Thermal Amorphization of Zeolitic Imidazolate Frameworks. Angew. Chemie Int. Ed. 50, 3067-3071 (2011).

36. Bennett, T. D. et al. Melt-Quenched Glasses of Metal-Organic Frameworks. J. Am. Chem. Soc. 138, 3484-3492 (2016).

37. Gaillac, R. et al. Liquid metal-organic frameworks. Nat Mater 16, 1149-1154 (2017).

38. Bennett, T. D. et al. Hybrid glasses from strong and fragile metal-organic framework liquids. Nat. Commun. 6, 8079 (2015).

39. Qiao, A. et al. A metal-organic framework with ultrahigh glass-forming ability. Sci. Adv. 4, (2018).

40. Zhou, C. et al. Metal-Organic Framework Glasses with Permanent Accessible Porosity. (2018). doi:10.26434/chemrxiv.6723473 
41. Wenqian, C. et al. Glass Formation of a Coordination Polymer Crystal for Enhanced Proton Conductivity and Material Flexibility. Angew. Chemie Int. Ed. $55,5195-5200(2016)$.

42. Kniep, R., Mootz, D. \& Vegas, A. Variscite. Acta Crystallogr. Sect. B Struct. Crystallogr. Cryst. Chem. 33, 263-265 (1977).

43. Song, Y., Zavalij, P. Y., Suzuki, M. \& Whittingham, M. S. New Iron(III) Phosphate Phases: Crystal Structure and Electrochemical and Magnetic Properties. Inorg. Chem. 41, 5778-5786 (2002).

44. Deiseroth, H. J. \& Müller -Buschbaum, H. Über Erdalkalimetalloxogallate. III. Untersuchung des Aufbaus von CaGa2O4. Zeitschrift für Anorg. und Allg. Chemie 396, 157-164 (1973).

45. Lazić, B., Kahlenberg, V. \& Konzett, J. Rietveld Analysis of a High Pressure Modification of Monocalcium Oxogallate (CaGa2O4). Zeitschrift für Anorg. und Allg. Chemie 631, 2411-2415 (2005).

46. Bennett, T. D. et al. Reversible pressure-induced amorphization of a zeolitic imidazolate framework (ZIF-4). Chem. Commun. 47, 7983-7985 (2011).

47. du Bourg, L. B., Ortiz, A. U., Boutin, A. \& Coudert, F. X. Thermal and mechanical stability of zeolitic imidazolate frameworks polymorphs. Apl Mater. 2, $124110(2014)$

48. Yang, S. et al. Cation-induced kinetic trapping and enhanced hydrogen adsorption in a modulated anionic metal-organic framework. Nat. Chem. 1, 487 (2009).

49. Thornton, A. W. et al. Porosity in metal-organic framework glasses. Chem. Commun. 52, 3750-3753 (2016).

50. Ortiz, A. U., Boutin, A., Fuchs, A. H. \& Coudert, F.-X. Investigating the 
Pressure-Induced Amorphization of Zeolitic Imidazolate Framework ZIF-8:

Mechanical Instability Due to Shear Mode Softening. J. Phys. Chem. Lett. 4, $1861-1865(2013)$.

51. Greaves, G. N. et al. Zeolite collapse and polyamorphism. J. Phys. Condens. Matter 19, 415102 (2007).

52. Meneau, N. G. and F. Probing the dynamics of instability in zeolitic materials. J. Phys. Condens. Matter 16, S3459 (2004).

53. Armand, M., Endres, F., MacFarlane, D. R., Ohno, H. \& Scrosati, B. Ionicliquid materials for the electrochemical challenges of the future. in Materials for Sustainable Energy 129-137 (Co-Published with Macmillan Publishers Ltd, UK, 2010). doi:doi:10.1142/9789814317665_0020

54. Lima, T. A., Paschoal, V. H., Faria, L. F. O. \& Ribeiro, M. C. C. Unraveling the Stepwise Melting of an Ionic Liquid. J. Phys. Chem. B 121, 4650-4655 (2017).

55. Mcgreevy, R. L. \& Pusztai, L. The Structure of Molten Salts. Source Proc. Math. Phys. Sci. 430, 241-261 (1990).

56. Yuan, S. et al. PCN-250 under Pressure: Sequential Phase Transformation and the Implications for MOF Densification. Joule 1, 806-815 (2017).

57. Mączka, M. et al. Temperature- and Pressure-Induced Phase Transitions in the Metal Formate Framework of [ND4][Zn(DCOO)3] and [NH4][Zn(HCOO)3]. Inorg. Chem. 53, 9615-9624 (2014).

58. Gustafsson, M. \& Zou, X. Crystal formation and size control of zeolitic imidazolate frameworks with mixed imidazolate linkers. J. Porous Mater. 20, 55-63 (2013).

59. Filik, J. et al. Processing two-dimensional X-ray diffraction and small-angle scattering data in DAWN 2. J. Appl. Crystallogr. 50, 959-966 (2017). 
60. Dorogokupets, P. I. \& Dewaele, A. Equations of state of MgO, Au, Pt, NaCl-B1, and $\mathrm{NaCl}-\mathrm{B} 2:$ Internally consistent high-temperature pressure scales. High Press. Res. 27, 431-446 (2007).

61. Dewaele, A., Torrent, M., Loubeyre, P. \& Mezouar, M. Compression curves of transition metals in the Mbar range: Experiments and projector augmentedwave calculations. Phys. Rev. B 78, 104102 (2008).

62. Rekhi, S., Dubrovinsky, L. S. \& Saxena, S. K. Temperature-induced ruby fluorescence shifts up to a pressure of $15 \mathrm{GPa}$ in an externally heated diamond anvil cell. HIGH Temp. Press. 31, 299-305 (1999).

63. Coelho, A. A. TOPAS and TOPAS-Academic: an optimization program integrating computer algebra and crystallographic objects written in C plus. J. Appl. Crystallogr. 51, 210-218 (2018). 